\title{
Novel approaches for large-scale two- dimensional hydrodynamic modelling of rivers
}

\author{
Mohamed F.M. Yossef ${ }^{1}, J . S$. de Jong ${ }^{1}$, A. Spruyt ${ }^{1}$, and M. Scholten ${ }^{2}$ \\ ${ }^{1}$ Deltares, Delft, the Netherlands - www.deltares.nl \\ ${ }^{2}$ Rijkswaterstaat, Lelystad, the Netherlands
}

\begin{abstract}
For decades, the decision-making process for water management in the Netherlands makes full utilisation of state of the art models. For rivers, two-dimensional hydrodynamic models are considered essential for a wide range of questions. Every five years, there is a major model revision that includes software updates, improved physical processes, new modelling strategy, and a new calibration. 2017 marked the setup and calibration of the first river model in the sixth generation of these models. In this paper, we discuss the most recent developments in twodimensional hydrodynamic modelling of rivers. We give an overview of the process followed to agree on the functional design of the model and address the use of the recently developed Delft3D Flexible Mesh suite. We address, in some details: i) a mesh independent approach for model setup; ii) the utilisation of a new calibration technique, which is automated using data assimilation and includes spatial and discharge dependencies; and iii) the use of a novel operational module to control hydraulic structures. The first river model within the 6th generation of models is that of the Meuse River, where the new approaches are being successfully applied. In conclusion: the mesh independent modelling approach offers great flexibility and facilitates that the same data set can be used for multiple versions of the model (e.g. different grid resolution; or different model extent). The automated calibration approach makes it possible to utilise a comprehensive calibration data set for a large-scale model in a reproducible way. The increased complexity of modelling has become possible over the last decade due to the availability of large datasets and increased computational power. This paper is particularly relevant for modellers and decision makers alike.
\end{abstract}

\section{Introduction}

The Netherlands is world renowned for its struggle with water. Over centuries, a sophisticated approach for water management has been developed. Since decades, the decision making process for water management questions, makes full utilisation of state of the art models. Particularly for rivers, there are a large number of models in place. Twodimensional morphodynamic models are used for river engineering and navigability studies $[1,2]$. One-dimensional models are mostly used for operational systems including early warning. Two-dimensional hydrodynamic models are considered essential for a wide range of questions, e.g. flood safety, water quality, infrastructure development, etc. These models 


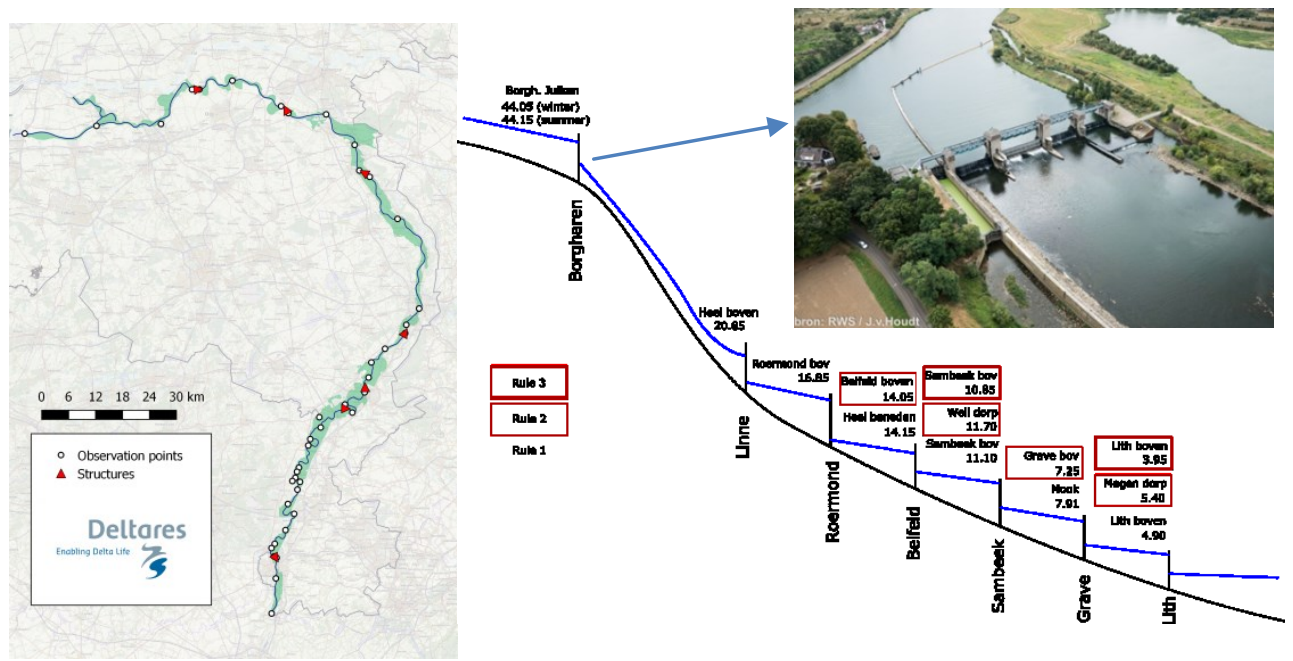

Fig. 1 The Meuse River in the Netherlands (left); and a schematic representation of the longitudinal profile showing the control structures and their operational rules (right).

are regularly updated. A yearly update cycle involves updating the bed levels, floodplain vegetation, and any changes in schematisations. Every five years, there is a major model revision that includes software updates, improved physical processes, new modelling strategy, and a new calibration. The year 2017 marked the setup and calibration of the first river model in the sixth generation of these models. For this paper, we use the model of the Meuse River to demonstrate the new approaches for model setup and calibration.

\subsection{The Meuse River}

The Meuse is a rain-dominated river with a total length of $925 \mathrm{~km}$, which originates in France and flows through Wallonia into the Netherlands at Eijsden. It usually floods during the winter with a combination of long-term rainfall, low evaporation, and snow melting. In 1993 a discharge of $3000 \mathrm{~m}^{3} / \mathrm{s}$ has been observed. Estimates of extreme flows are made in the frame work of the GRADE project [3]; discharges of $4100 \mathrm{~m}^{3} / \mathrm{s}(\mathrm{T}=1 / 3000)$ to $5000(\mathrm{~T}$ $=1 / 22500) \mathrm{m}^{3} / \mathrm{s}$ are used to evaluate flood risks in the Netherlands. The Meuse regularly experiences periods of rather low discharge, which motivated the 1994 agreement on the protection of the Meuse. In the Netherlands, the Meuse flows for some $250 \mathrm{~km}$ and merges with the Rhine before discharging into the North Sea. Along its course, there are 7 structures (see Fig. 1), with the primary objective to regulate the water levels for navigation purposes; they are fully opened during floods.

\subsection{Model functional design - process and requirements}

The basic idea for the (new) $6^{\text {th }}$ generation models is: to set up an integrated model for the complete main Dutch water system. The design and settings of the models must allow a wide range of applications, both in type of water system (Viz. rivers, lakes, and sea) and processes (e.g. hydrodynamics and constituents). Also coupling should be possible with other modules or models (e.g. waves, morphology, water quality, meteorology); see Fig. 2. For this, generic functional and technical specifications were defined in coordination with groups of experts (with system knowledge, modelling, and software), via several workshops. The models are firstly made for separate water bodies mainly focussing on 
hydrodynamics in $2 \mathrm{D}$, with the idea that more processes will be incorporated and the models will be connected in a later stage.

A number of workshops were organised to reach a consensus on the functional design the models. The processes started by making an inventory of the function of each waterbody, the corresponding management questions, expectations from the model, and to set the priorities between conflicting demands. This led to the definition of broad design criteria, which was detailed in a next stage. These criteria covered aspect such as: model dimensions (1D, 2D, 3D), mesh coverage and its resolution, timeframe of expected

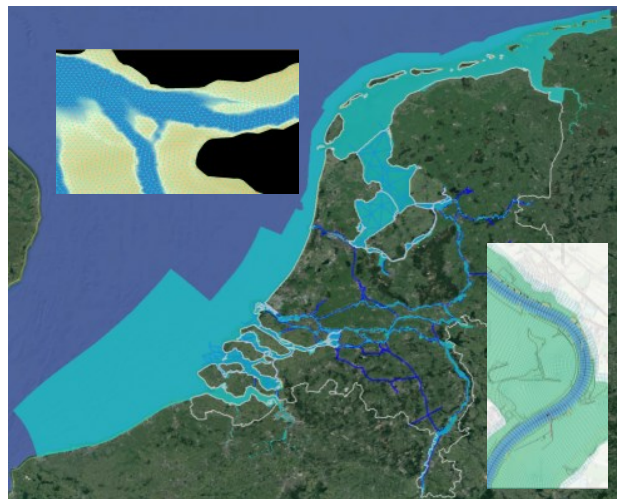

Fig. 2 Map of the Netherlands showing the different models and example mesh(s). simulations (from short term to long term), type of boundary conditions and forcing, calibration and validation acceptance criteria, and desired computational time. To meet all demands, a number of models for every waterbody were agreed and priority of execution was set; e.g. 1D-models for water allocation, 2Dmodels: for flood safety and permits, and 3D-models for detailed studies around structures. The highest priority was identified for flood safety and issuing permits for works on floodplains. On a parallel track, pilot studies were executed to identify unified model parameter settings and schematisation options that allow seamless integration between the different models in a later stage.

In this paper, we use the model of the Meuse River to demonstrate the most recent developments in the setup and calibration of large-scale river hydraulic models. The model covers the Meuse model in the Netherlands and can run for relatively long time series; the same model is planned to be extended to include morphology, which may run for several decades [e.g.1, 2]. The model is made using the open source Delft3D FM Software Suite, which integrates multi-dimensional approaches and hydrodynamics, morphodynamics, water quality and waves in single package [4]. Herein, we focus on two-dimensional hydrodynamic modelling of rivers.

\section{Model setup for the Meuse River}

\subsection{Data sets}

Successful modelling requires three different groups of data sets: i) model construction data set, ii) calibration and validation data set, and iii) scenario analysis data set. The model construction data set includes all geo-data that allows for a successful schematisation. A successful schematisation may be defined as sufficiently accurate projection of the prototype features (land-boundaries, topography, vegetation, structures, etc.) on the mesh such that the model is fit-for-purpose. The calibration and validation data sets, includes all forcings (discharges, wind, etc.) and observations (water levels, velocities, etc.) in a relatively narrow window of time (calibration \& validation periods) with high frequency and high accuracy. The scenario analysis data set is often a derived set based on long-term observations or future projections (historical data, design conditions, etc.).

For all water bodies in the Netherlands including floodplains, the geometrical and vegetation coverage data is managed in a GIS-database that is model independent. The historical data sets (short and long terms) are managed by RWS. As forcings, we used 
discharges in the upstream boundary and 27 laterals, and downstream water levels. We will discuss additional data when relevant.

\subsection{The mesh}

Delft3D FM Software Suite employs a flexible mesh approach, which allows combinations of mesh elements that ranges from tringles to hexagons. For river models, it is of vital importance that the mesh follows the river backlines. This is relatively straightforward for detailed models; but for large-scale models, it puts high pressure on generating an efficient and high-quality mesh. The model functional design criteria specified that the number of cells within the main channel must be at least 8 cells of at most $20 \mathrm{~m}$ wide in the transverse direction. In the floodplains, cell dimensions can reach up to $40 \mathrm{~m}$. These criteria lead to a model with 340.347 cells, and 774.927 flow links; Fig. 3 shows an example of the mesh.

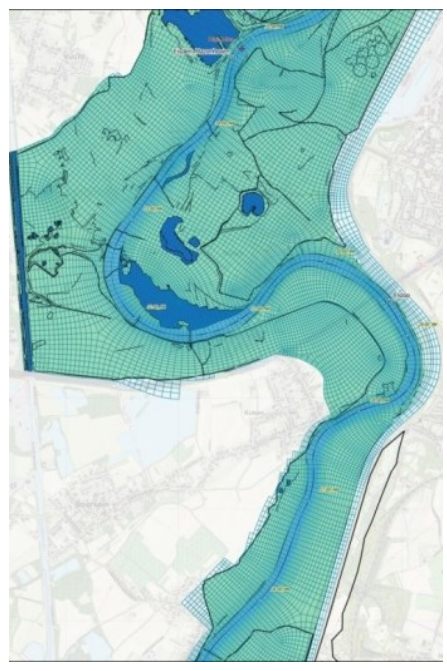

Fig. 3 Mesh for the Meuse River near Elsloo

For an efficient computational mesh, we give a preference for quadrangular cells over triangular, pentagons, or hexagons. In addition, the mesh is aligned with the main channel of the river for efficient modelling of low flows and aligned, as much as possible, with the streamlines for flood conditions. These choices are particularly important for large-scale models, and they offer the following advantages: i) less numerical diffusion due to alignment with the flow direction; ii) relatively large calculation time step is possible due to the larger volume of quadrilaterals over triangles; iii) less cells are needed due to the better mesh convergence of quadrangles. However, the design of such a mesh is rather labour intensive and requirements for mesh orthogonality and smoothness are harder to achieve. Still, for a model which that is intended for use for several years, the effort is worth it.

For the generation of the mesh, we used a multiblock structured approach [see e.g. 5], in which different curvilinear blocks are connected to each other by the unstructured administration. Mesh orthogonality is kept lower than 0.01 except for some locations near boundaries or outside flow area where it reached 0.02 .

\subsection{Mesh independent modelling approach}

Rijkswaterstaat manages the geographical information for all waterbodies in a GIS databases. In this database, data from surveys, the digital terrain file, design drawings, vegetation coverage, structures, and many other data sources are combined. The database is then suitable for projection on the mesh of the model.

The procedures are made with the help of a dedicated ArcGIS extension (Baseline) and a geographical database, intended for the storage, adaptation and presentation of river data. Baseline automatically generates various input files ready for use in the model. Thus, it guarantees that the model schematisations are reproducible and mesh independent. Using these procedures, we generated six different models for the years: 1993, 1995, 2011, 2014, 2017 , and a model for the future situation including planned measures.

We emphasise the importance of proper schematisations of the topography; viz. the projection of bed levels on the mesh. Delft3D FM Suite employs different approaches for schematisation of bed level (see Fig. 4 \& Fig. 5). After multiple tests, and by giving more importance to accurate computation of velocity (momentum), we select a schematisation similar to Fig. $4 b$; it yields more the accurate representation of the depth at velocity points. 


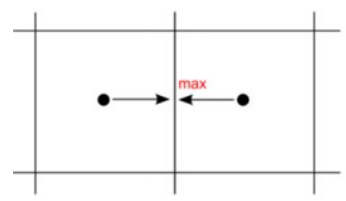

(a)

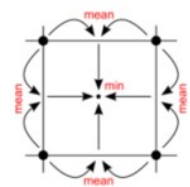

(b)

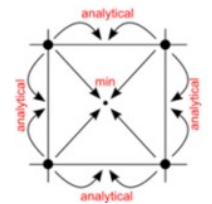

(c)

bed level type 1

bed level type 3 , convayence $2 \mathrm{D}=-1 \quad$ bed level type 3 , convayence $2 \mathrm{D} 1 \geq 1$

Fig. 4 Different approaches for bed schematisations (a) bed level defined in cell centre \& deduced at velocity point, (b \& c) bed level defined in cell corner \& deduced at velocity and water level points.

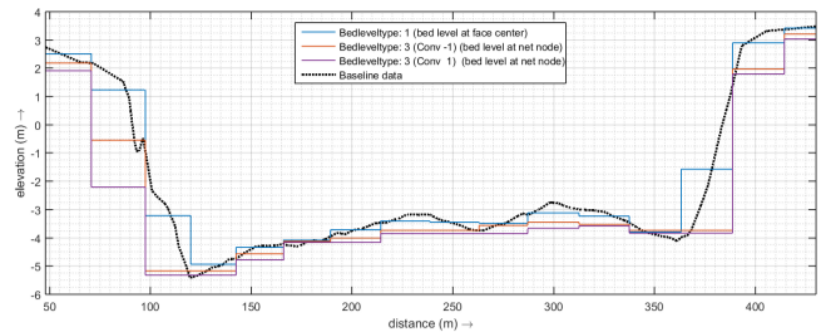

Fig. 5 Difference between bed elevation of the prototype and different types of bed schematisations.

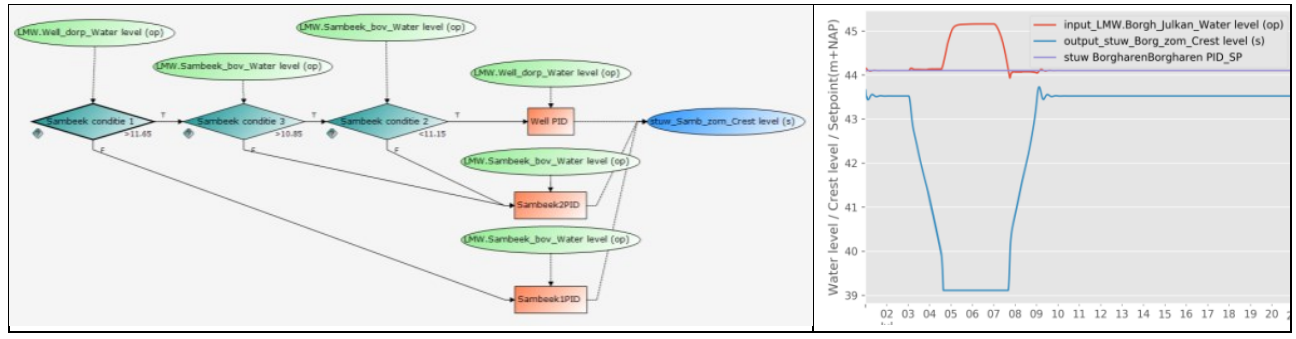

Fig. 6 Diagram showing the operational rules for the structure at Sambeek (left) and resulting model calculated gate level and water levels (right).

\subsection{Hydraulic structures}

As demonstrated in Fig. 1(right), the Meuse River includes multiple control structures. The operation of these structures is rather complex as it involves a combination of the discharge stage, the water levels, the method to open and close gates. The exact reproduction of how a structure is operated is very difficult as it involves a combination of hydraulic conditions as well as the decision of the operator. In addition, the individual gates have smaller resolution than the computational mesh. Accordingly, it has decided to model the structure such that the model replicates the behaviour as much as possible rather than to model actual geometry.

For control, a feedback control has been implemented in which the desired operational water level is checked with the help of a PID controller. The objective of the PID controller is to set the water levels upstream of the weir as close as possible to measurements during calibration stage; and to match the set water level during operation. The PID takes the form:

$$
w_{i}=w_{i-1}+k_{p} e(t)+k_{i} \int_{0}^{t} s(t) d t+k_{d} \frac{d e(t)}{d t}
$$

where $w$ is gate level, $i$ time step, $k_{p}, k_{i} \& k_{d}=$ proportionality, integral, and differential constants for PID controller, and $e=$ error in water level, $t$ for time [6]. The constants are subject to calibration. Fig. 6 shows the set-up of the operational rules for the structure at Sambeek and the resulting gate and water levels, showing a good performance. 


\subsection{Definition of bed roughness}

Until the $5^{\text {th }}$ generation model, and similar to conventional calibration approach of hydraulic models, the roughness coefficient(s) is used as the primary calibration parameter. Accordingly, the definition of an initial roughness value is not critical as it will be changed during the calibration step. However, for $6^{\text {th }}$ generation models a new approach has been developed to allow separation between the definition of roughness as a physically-based parameter and calibration factor as a model requirement to compensate for any uncertainty or inaccuracy in model schematization (including roughness).

The physically-based roughness is the basis of the calibration and is chosen based on physical properties of each river reach. For the floodplain, a complex definition based on vegetation coverage is extracted from the GIS-database. The vegetation field is converted into roughness based on a sub-grid approach (see Trachytopes in [7]). For the main channel, it is based on the sediment characteristics and variability of bed forms. In this regard, the Meuse can be divided in two reaches; the gravel-bed GrindMaas and the sandbed ZandMaas. For the GrindMaas, upstream of the structure at Linne, there are no dunes. Accordingly, we use a constant White-Colebrook roughness of $k=0.3 \mathrm{~m}$.

Downstream of Linne, the effect of dunes must be included in the definition of roughness. Accordingly a roughness predictor based on dune height is used. Information about the dune heights and sediment characteristics of the Meuse are scarce; for that we use the data of Julien \& Klaasen (1995) and Frings $(2002)$ [8, 9]. Then, the roughness can be estimated based on the on the formula of Van Rijn [10]. The result is compared with analytical values based on the simplified formula of Van Rijn (Eq. 2). The comparison showed that values $\mathrm{A}=0.1 \& \mathrm{~B}=2.5$ are acceptable approximation.

$$
k=A h^{0.7}\left(1-e^{-B h^{-0.3}}\right)
$$

\section{Calibration and validation}

\subsection{Calibration approach}

The primary objective of the calibration of a hydrodynamic model is to ensure that it can reproduce the water levels at measurement stations; in case there is more than one branch, the discharge distribution is as important. Calibration is performed based on the application of discharge dependant spatially varying calibration factor. As an intermediate step, all roughness types used in the schematisation are converted to a unified user-defined type (Manning, herein). Afterwards, the calibration factor is multiplied by the previously defined physically-based roughness. For the Meuse model, we use 32 calibration reaches (between measuring stations) and 5 discharge levels. Spatial transition, between reaches, is smooth to guarantee that the model is suitable to be extended for morphology.

When there is wealth of data, and the demands on the model are as high as in our case, the selection of calibration and validation periods becomes important. Domhof et al. [11] shows that the selection of calibration discharge stages affects the model result. Calibration discharge levels can be selected based on: return period, geometry, available data, and largest error in model results. With a priority as listed, we select calibration and validation periods as in Table 1. Accordingly, four different model schematisations were used.

Table 1 Calibration and validation discharge conditions and corresponding periods

\begin{tabular}{|l|c|c|c|c|c|c|c|c|c|c|}
\hline Data set for schematisations & \multicolumn{7}{|c|}{ Discharge levels for calibration (K) \& validation (V) runs } \\
\cline { 2 - 12 } \& boundary conditions & $100 \mathrm{~m}^{3} / \mathrm{s}$ & $800 \mathrm{~m}^{3} / \mathrm{s}$ & $1700 \mathrm{~m}^{3} / \mathrm{s}$ & $2300 \mathrm{~m}^{3} / \mathrm{s}$ & $2700 \mathrm{~m}^{3} / \mathrm{s}$ \\
\hline $01 / 11-1993-01 / 02 / 1994$ & & & & & & & & $\mathbf{V}$ & & $\mathbf{V}$ \\
\hline $01 / 12 / 1994-01 / 03 / 1995$ & & & & & & $\mathbf{V}$ & & & $\mathbf{K}$ & \\
\hline $01 / 11 / 2010-01 / 02 / 2011$ & & & & & $\mathbf{K}$ & & $\mathbf{K}$ & & & \\
\hline $01 / 07 / 2014-01 / 07 / 2015$ & $\mathbf{K}$ & $\mathbf{V}$ & $\mathbf{K}$ & $\mathbf{V}$ & & & & & & \\
\hline
\end{tabular}




\subsection{Automation of the calibration process - OpenDA}

Due to the large amount of data, and extent of the calibration periods and number of reaches to be calibrated, we use automated calibration procedure utilising the data assimilation tool OpenDA [12]. We use of the OpenDA DuD algorithm, which is based on a self-learning concept; in which, on the basis of input Predictors (water levels stations) and RegularisationConstants (calibration coefficient per reach), the correlation between the calibration reaches and measuring stations is found via a number of initialisation model runs; initialisation matrix is shown in Fig. 7a. Subsequently, an optimisation problem is solved to minimise the deviation between calculated and measured water levels; Fig. 7b shows the cost function, which rapidly reduces after the initialisation runs indicating rapid convergence. From Fig. 7a, we see a nearly diagonal matrix, which indicates that mostly, each calibration reach affects the result of observation point in its upstream. The deviation from perfect diagonal indicates that the effect of some calibration reaches extend to affect more observation points.

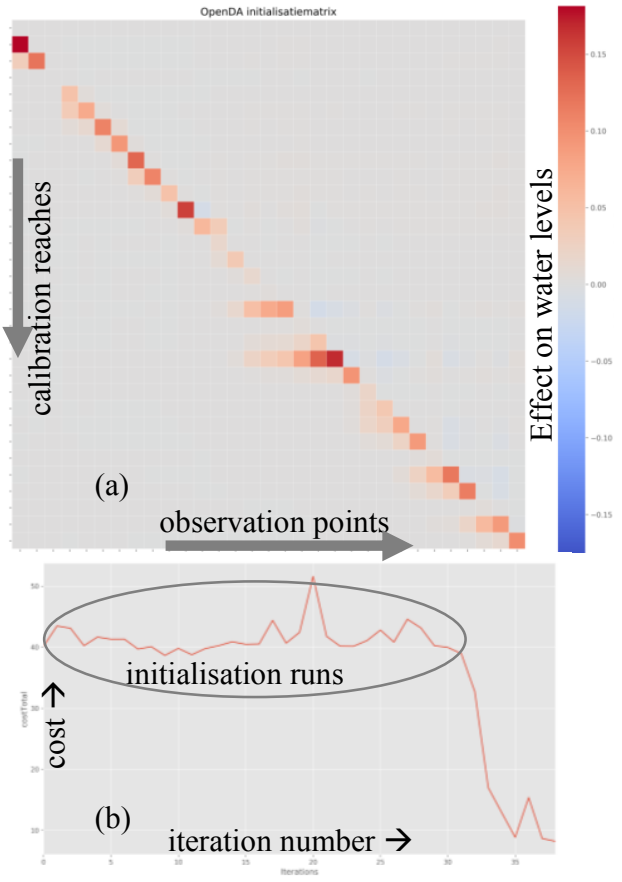

Fig. 7 Initialisation matrix (a) and value of cost function (b) - result of OpenDA

\section{Results}

The result of the calibration is a set of calibration factors, corresponding to each discharge levels and for every river reach. These are aggregated for use in future model applications. The calibration factors have an average value for the 5 discharge levels of $0.84,0.82,0.95$, 0.94 and 0.95 respectively. This differs slightly from 1.0 , which shows that the selected background roughness is good. However, a minimum of 0.30 and a maximum of 1.88 indicate that locally large errors, in schematisation or roughness, have to be corrected; particularly in the small reaches with structures. The overall result is shown in Fig. 8.

The result of one of the validation runs is shown in Fig. 9. It shows that the performance of the model is very good in a wide range of discharges (water levels after calibration have: Bias $=0.109 \mathrm{~m}, R M S E=0.164 \mathrm{~m} \&$ Sted $v=0.123 \mathrm{~m})$. The model has been successfully tested under design flood conditions and showed that it is robust and ready for application.

\section{Concluding remarks}

We presented some recent developments in the setup and calibration of hydrodynamic models; the $6^{\text {th }}$ generation model of the Meuse River was used to demonstrate these concepts. We demonstrated that, the mesh independent modelling approach offers great flexibility and facilitates that the same data set can be used for multiple versions of the model (e.g. different mesh resolution, different model extent). A PID controller is used to model the complex operational rules of structures. The calibration approach is robust and makes use of a discharge-dependant spatially varying calibration factor, which is separate 
from the physically-based roughness. The automated calibration approach makes it easy to utilise a comprehensive data set for a large-scale model in a reproducible way. Finally, the increased complexity of modelling has become possible over the last decade due to availability of large data sets, increased computational power. Such complexity aims to reducing the uncertainty of models and to facilitate a better decision making process.

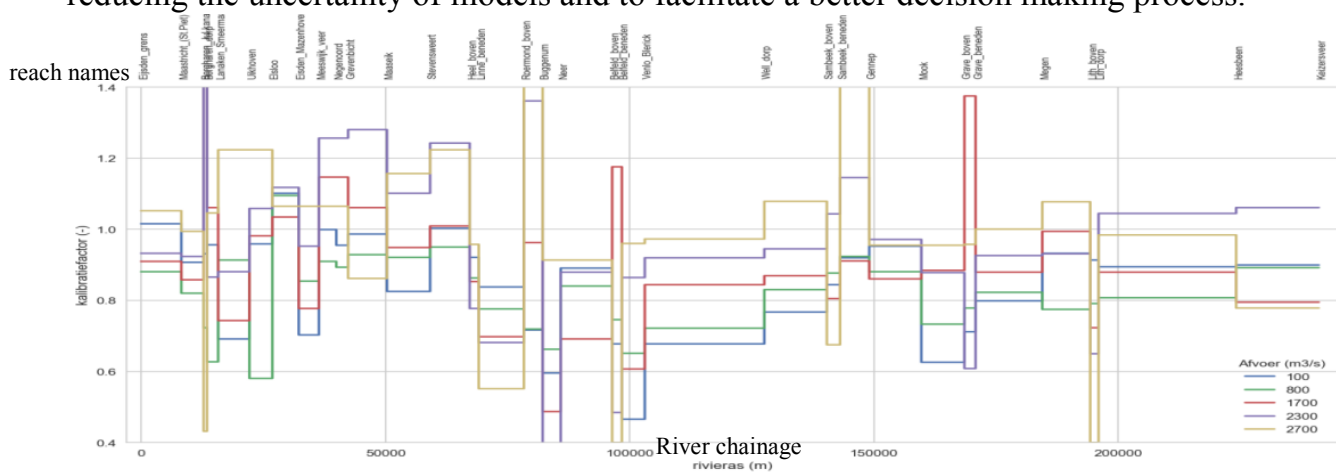

Fig. 8 Longitudinal profile shown calibration factor for the 5 discharge levels

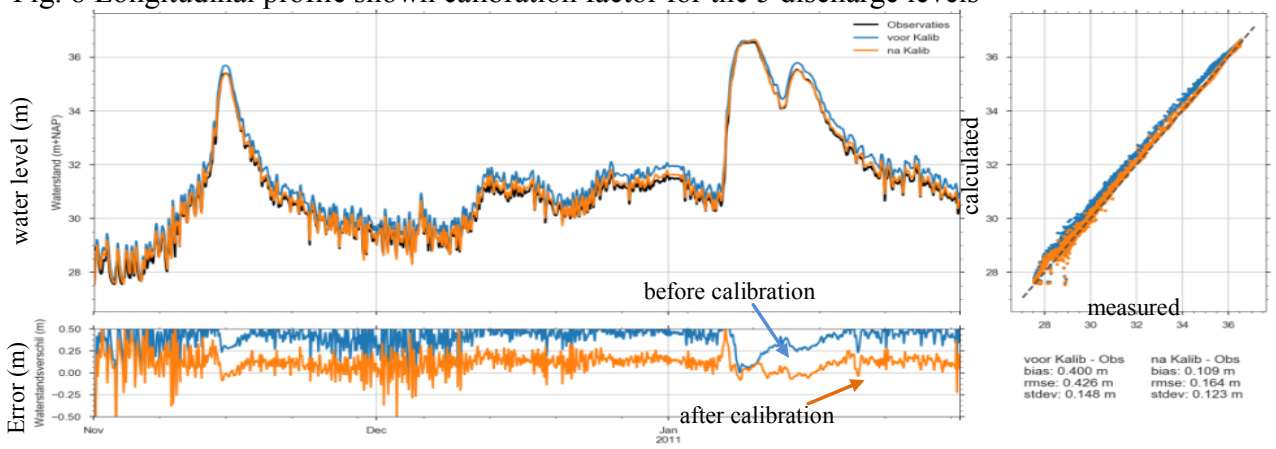

Fig. 9 water level time series - observations and model results before and after calibration (at Eisden)

\section{References}

1. Yossef, M.F.M., et al., Innovative techniques in modelling large-scale river morphology, in River Flow 2008, M. Altinakar, et al., 2008, Izmir, Turkey.

2. Yossef, M.F.M. and K. Sloff. Detailed Modelling of River Morphological Response to Climate Change Scenarios. in River Flow 2012. San José, Costa Rica

3. Hegnauer, M., et al., Generator of Rainfall and Discharge Extremes (GRADE) for the Rhine and Meuse basins - Final report of GRADE 2.0. 2014, Deltares: Delft.

4. Deltares. Delft3D Flexiable Mesh, open source, http://oss.deltares.nl/web/delft3dfm.

5. Armstrong, C.G., et al., Common themes in multi-block structured quad/hex mesh generation. Procedia Engineering, 2015. 124: p. 70-82.

6. Schwanenberg, D. and B. Becker, RTC-Tools - Software Tools for Modeling Real-Time Control https://oss.deltares.n1/documents/102774/467082/rtc-tools UserManual.pdf.

7. Deltares, Delft3D Flexible Mesh Suite, User Manual. 2018, https://content.oss.deltares.nl/delft3d/manuals/D-Flow_FM_User_Manual.pdf.

8. Frings, R., Duinen, hydraulische ruwheid en bodemtransport in het zomerbed van de Maas bij Broekhuizen. Universiteit Utrecht, ISBN 9077079033, 9789077079034 ICG 02/1.

9. Julien, P.Y. and G.J. Klaassen, Sand-dune geometry of large rivers during floods. Journal of Hydraulic Engineering, 1995. 121(9): p. 657-663.

10. Van Rijn, L.C., Sediment Transport, Part III: Bed Forms and Alluvial Roughness. Journal of Hydraulic Engineering, ASCE, 1984. 110(12): p. 1733-1754.

11. Domhof, B.C.A., et al., Discharge and location dependency of calibrated main channel roughness - Case study on the River Waal, in River Flow 2018. 2018: Lyon, France.

12. OpenDA: https://www.openda.org. 